\title{
Variations
}

Variations

Revue internationale de théorie critique

$22 \mid 2019$

Gorz, l'intempestif

\section{Table ronde au sujet du livre Après Habermas à l'Université de Liège - Humanités critiques}

Caroline Glorie, Jeremy Hamers, Timothée Moreau et Alexander Neumann

\section{(2) OpenEdition}

Journals

Édition électronique

URL : http://journals.openedition.org/variations/1071

DOI : 10.4000/variations. 1071

ISSN : 1968-3960

Éditeur

Les amis de Variations

Référence électronique

Caroline Glorie, Jeremy Hamers, Timothée Moreau et Alexander Neumann, « Table ronde au sujet du livre Après Habermas à l'Université de Liège - Humanités critiques », Variations [En ligne], 22 | 2019, mis en ligne le 04 mars 2019, consulté le 01 mai 2019. URL : http://journals.openedition.org/ variations/1071; DOI : 10.4000/variations. 1071

Ce document a été généré automatiquement le 1 mai 2019.

Les ami•e•s de Variations 


\title{
Table ronde au sujet du livre Après Habermas à l'Université de Liège - Humanités critiques
}

\author{
Caroline Glorie, Jeremy Hamers, Timothée Moreau et Alexander \\ Neumann
}

\section{Jeremy Hamers}

1 Cher Alexander Neumann, je voudrais tout d'abord vous remercier d'avoir accepté notre invitation à vous entretenir ici à l'Université de Liège avec le groupe de recherche ARC GENACH - Genèse et actualités des humanités critiques - France-Allemagne - 1945-1980 - au sujet de votre ouvrage Après Habermas. La Théorie critique n'a pas dit son dernier mot. ${ }^{1}$

2 Admettons-le d'entrée de jeu: le titre et le sous-titre de votre ouvrage sont faits pour nous plaire, a priori. Pour deux raisons au moins qui se cristallisent, pour la première dans le titre de votre livre, pour la seconde dans son sous-titre.

3 Au gré de nos lectures et de nos travaux tout d'abord, nous avons parfois l'impression que Habermas, et à sa suite Honneth, monopolisent quelque peu l'attention des études sur les héritages de la Théorie critique. Or, nous ne nous reconnaissons pas dans Habermas, ou en tout cas beaucoup moins que dans d'autres héritages intellectuels de l'École de Francfort que nous avons commencé à explorer il y a quelques années. Je songe notamment à Alexander Kluge et à Oskar Negt, mais aussi à Hans Magnus Enzensberger. Ils ont tous trois dynamisé le legs francfortois, en jouant notamment de la rupture et du " pas de côté » que vous synthétisez dans la dernière partie de votre ouvrage en évoquant une « créolisation inaboutie ${ }^{2}$ ». Il nous semble que ces auteurs, en tant qu'ils sont, eux aussi, des héritiers de l'École de Francfort, nous engagent dans un dialogue - au moins aussi fécond que dans le cas de Habermas - avec la Théorie critique, mais aussi avec une certaine pensée contemporaine française (Sartre, Foucault, et alli) ou des penseurs que l'on considère parfois comme pré-francfortois ou en marge de la Théorie critique (Hannah Arendt, Walter Benjamin, et alli). 
Ensuite, le sous-titre de votre livre nous a également séduits d'entrée de jeu car si nous travaillons aujourd'hui ensemble à l'exploration de plusieurs théories critiques au carrefour de la France et de l'Allemagne, c'est bien parce que la Théorie critique est marquée à nos yeux par une absolue actualité. Cette Théorie critique n'a pas dit son dernier mot en effet, quand bien même certains auteurs contemporains tentent de la disqualifier et d'en proclamer l'irréductible obsolescence.

Partant de cette sympathie a priori pour votre travail de relecture de l'École de Francfort et de ses héritages, l'actualité de la Théorie critique, c'est-à-dire sa capacité à faire retour, me permet de vous adresser deux remarques introductives.

6 Une première impression générale nous gagne à la lecture de votre texte : il nous semble que dès l'entame de votre ouvrage, vous ne "faites pas toujours dans le détail ». Vous écrivez de façon totalement libre, en avançant parfois très vite, et en vous autorisant à produire une série de jugements. Il suffit, pour s'en rendre compte, de lire les premières pages de votre livre qui adoptent les traits d'une attaque en règle contre Habermas. Cette liberté est certes séduisante. Mais elle nous perd aussi quelque peu, flirte avec l'essai, voire avec le texte polémique.

La seconde remarque introductive que je voudrais vous adresser fait également fond sur une impression générale ambivalente. La Théorie critique est parfois contestée aujourd'hui en tant qu'elle ne serait pas ou plus pertinente au contact de nos phénomènes, productions et dispositifs contemporains ${ }^{3}$. Cette façon d'éprouver la pertinence des pensées d'Adorno ou de Horkheimer en les croisant avec un ensemble d'objets empiriques relève à nos yeux d'une erreur de méthode. La Théorie critique ne se mesure pas au réel, pas plus qu'elle ne prétend mesurer le réel. Pour autant, comme vous le rappelez dans votre ouvrage, elle ne parie pas non plus sur une fracture volontaire entre raison et réalité pour se mettre à l'abri des fausses identifications que la seconde offrirait en permanence au sujet pensant ${ }^{4}$. Nous pensons au contraire que le rapport de la Théorie critique au réel, à une réalité sociale donnée, est le plus fécond lorsqu'il peut être pensé sur le mode de l'invention fictionnelle, une invention qui affecte précisément le rapport entre théorie et réalité et qui en problématise dès lors les conditions d'existence et de pertinence dans un contexte donné. C'est ce que nous appelons au sein du groupe de recherche ici réuni un « savoir critique ${ }^{5}$ ». Dans le débat opposant sociologie de Francfort et empirie anglo-saxonne des études en sciences sociales, Adorno s'est défendu lui-même de ce malentendu sur les missions de la Théorie critique. Il s'agissait en effet pour lui d'éviter l'utilitarisme tout autant que le cynisme de sa discipline. Et dans votre ouvrage, vous rappelez vous-même à plusieurs reprises à quel point l'empirie sociologique peut être dangereuse. Or, il nous semble parfois que votre opération de déconstruction de la place occupée par Habermas recourt précisément à la vérification de la théorie par un recoupement avec le réel. Vous affirmez par exemple à plusieurs reprises que tel ou tel événement donne tort à Habermas. Je songe notamment aux conséquences de la crise de 2007-2008 qui, dites-vous, a "montré que Habermas s'était trompé ». Ailleurs vous vous faites encore le relais de la critique des catégories idéal-typiques habermassiennes par Negt au nom du fait que leur fondement historique escamote le monde d'un pan entier de sa réalité sociale. En somme, que cela soit sous votre plume ou sous celle d'autres penseurs auxquels vous tenez, n'est-ce pas toujours le réel qui disqualifie Habermas? Et, si ce raccourci m'est permis, n'est-ce pas, peut-être, le signe que sa pensée ne constitue pas une théorie ou un savoir critique? 


\section{Alexander Neumann}

8 Merci d'abord de votre invitation chaleureuse et de la forme très ouverte et vivante qui s'offre ici à la discussion. Si le style de mon écriture vous a troublé, c'est sans doute bon signe. L'éditeur, Aymeric Monville, qui compte dans son catalogue des classiques comme George Lukacs ou Lucien Goldmann, m'avait demandé après lecture si j'avais vraiment écrit directement en français, alors que mon nom indique que j'ai grandi plus à l'Est. Il est vrai que je n'ai pas cherché à me limiter à une démonstration académique d'un genre scolaire, surtout pour ce chapitre introductif qui veut exposer les aspects problématiques du discours de Habermas, qui se situe loin de la Théorie critique. Mon livre n'est pas un essai, il se fonde sur une habilitation à diriger des recherches sur le rapport de la sociologie à la Théorie critique. Cette construction comporte un côté cumulatif ou plutôt une forme de montage, qui regroupe un ensemble de réflexions développées depuis une vingtaine d'années. Il ne s'agit pas pour moi d'épouser le réel, de le répéter d'une manière purement descriptive ou positiviste. Cela ne veut pas dire que la Théorie critique ne passe pas par l'épreuve du réel, où qu'elle se situerait en dehors de l'expérience sociale et historique, bien au contraire.

9 Pour ce livre, comme pour d'autres, je prends le pari de voir qui cela peut bien intéresser, parmi les éditeurs ou lectrices et lecteurs, sans me limiter aux seuls spécialistes, ce qui a plutôt bien marché. Certains lecteurs ont trouvé le corps du livre plutôt dense, sinon ardu, car c'est très documenté sur le plan scientifique et parfois dense du point de vue conceptuel. Dans une recension, Mikael Chambru trouve qu'il s'agit d'une contribution essentielle aux recherches interrogeant le concept d'espace public. Oskar Negt l'a évalué dans sa forme scientifique initiale, se demandant si cela n'augurait pas un opus magnum, et il a salué le résultat livresque.

10 Même si ce n'est pas la forme de ce texte, je suis très sensible à l'essai, ceux que j'ai aimé et cité dans le livre concernent Montaigne, le texte sur l'humanisme existentialiste de Sartre, le Paris, Capitale de Benjamin, la théorie féministe King Kong de Despentes, le Faust iconoclaste d'Oskar Negt, et surtout les Lipstick Traces de Greil Marcus, une sorte d'histoire secrète de la Théorie critique au 20ème siècle.

11 Walter Benjamin, que vous venez d'évoquer, me parle particulièrement. Il ne se situe pas en marge, mais au coeur de la Théorie critque, il participe directement de sa formulation, comme le montrent les archives Adorno et Benjamin à l'Académie des sciences de Berlin, lorsqu'il intervient par exemple dans la formulation de La dialectique de la raison, à partir de ses Thèses sur le concept d'Histoire. Benjamin a aussi écrit une habilitation à diriger des recherches sur le baroque, appuyée par Adorno, et qui ne fut jamais reconnue sur le plan académique, mais sa principale contribution se présente sous la forme d'essais aussi lumineux que décifsifs. Adorno a ensuite conceptualisé le caractère émancipateur de l'essai comme forme. En réalité, les auteurs dont on parle, Benjamin, Horkheimer, Adorno, ou encore Negt, ont cultivé un style très concis sur le plan conceptuel, sans renoncer à une forme de distanciation ironique, par exemple lorsque Adorno choisit le titre Minima moralia en lieu et place des Maxima moralia, ou quand Negt et Kluge décalent le titre de Lukacs Histoire et conscience de classe pour en faire leur livre Histoire et subjectivité rebelle. Le concept de Kulturindustrie en témoigne aussi, ce concept est une contradiction dans les termes dont l'implication ironique est claire en allemand, car l'industrie ne produit pas la culture, jamais de la vie. J'ai moi-même repris un titre français d'Habermas, Après Marx, pour en faire Après Habermas, poursuivant par là une certaine filiation 
dialectique. Le fait ne vous a pas échappé que je n'ai pas écrit un livre sur Habermas, mais sur la Théorie critique actuelle, en dehors d'Habermas dont je ne fais qu'esquisser les positions en introduction, puis par rappels successifs, pour situer le débat dans sa globalité. Il est vrai que cette esquisse introductive assez libre est venue s'ajouter à la partie théorique de l'habilitation à diriger des recherches qui constitue le socle de mon livre, un peu comme la nouvelle introduction d'Habermas de 1990 est venue s'ajouter après-coup à son livre L'espace public, qui correspond à sa propre habilitation.

En dehors de ses livres les plus savants, Habermas a souvent versé dans la polémique ou même la saillie pamphlétaire, en traitant Dutschke de fasciste, Negt et Kluge d'auteurs surréalistes, Adorno d'utopiste, en appelant au bombardement de la Serbie en dehors du droit international, en prenant position contre le pacifisme de Kant pour actualiser la pensée de Carl Schmitt, en se querellant avec les néo-réactionnaires Nolte ou Sloterdijk, etc. C'est sur ce dernier aspect qu'Habermas a le mieux employé son talent polémique, à mon sens, au sens d'un art du débat, en débusquant tout le potentiel autoritaire qui est contenu dans Les règles pour un parc humain. Sloterdijk a précisé sa pensée, par la suite, dans le sens que Habermas craignait, en exigeant la supressions de toute forme d'allocation sociale pour les pauvres 'qui volent les riches', en alimentant le sentiment anti-immigrés, et en jouant sur des éléments de porpagande nazie lorsqu'il invente un éther du mensonge autour de la presse libérale, là où Goebbels avait vilipendé la presse $d u$ mensonge. La polémique littéraire a des vertus d'éclaircissement du débat de fond, et Habermas en use en tant qu'intellectuel politique, engagé. Hélas, il s'est littéralement laissé aveugler par le candidat présidentiel Macron, se disant lui-même 'ébloui' par le personnage dans un article pour l'Obs en novembre 2017. Le moment où le réel s'est chargé de contredire le plus nettement fut la crise capitaliste globale de 2008, qui a relancé un ensemble de thématiques du Capital de Marx, ce qu'aucun intellectuel de gauche conteste aujourd'hui, alors que Habermas avait théorisé dans les années 1970 l'idée que cette critique de l'économie politique ne pouvait plus rien nous apporter. Pour cette raison, Negt a qualifié la théorisation habermassienne des années 1980 de Zeitdiagnose, de diagnostic d'époque éronné. L'exigence de maintenir un rapport à l'expérience empirique fut d'ailleurs formulé par Habermas lui-même dans son livre Théorie und Praxis en 1976.

Oui, Habermas s'est souvent trompé, et vous vous trompez si vous pensez que ce constat s'accorde mal avec une démarche scientifique, ou si vous croyez que Habermas ne se trompe jamais. De nombreux livres académiques contiennent la phrase : Habermas se trompe..., faites un essai sur n'importe lequel moteur de recherche, vous le verrez aussitôt. Vous semblez craindre le jugement critique, mais notre faculté de porter des jugements fonde littéralement la critique en sciences humaines, depuis la Critique de Kant. L'idée même que des philosophes ou sociologues ne devraient pas porter de jugement me semble plutôt conformiste. Ne vous laissez pas intimider par la fausse traduction américaine et française du principe de Wertfreiheit, qui recommande la liberté, mais certainement pas une prétenue "neutralité axiologique". Dans le livre dont on parle, je cite Max Weber qui nous avait expressément averti que : la position médiane ne contient pas davantage une once de vérité que les idéaux partisans les plus extrêmes. ${ }^{7}$

14 La critique est une épreuve du réel; chez Kant c'est l'épreuve de la révolution française qui donne raison au droit naturel sur le droit divin, car l'existence de dieu ne peut pas être prouvé ontologiquement et philosophiquement. ${ }^{8}$ Et la Théorie critique nait à son tour de la révolution allemande des conseils de 1918, comme je l'ai rappelé dans le livre. 
En ce sens, l'élaboration de la Théorie critique, depuis l'entre deux guerres, n'a rien de fictionnel. Dire cela n'exprime pas un désaccord entre moi et votre groupe de recherche sur les humanités critiques, mais rappelle simplement un désaccord qui se fait jour entre la Théorie critique d'une part, et l'ontologie de facture heideggérienne de l'autre. C'est un désaccord qui est souligné par Benjamin dès 1935 dans ses écrits parisiens, avant d'être explicitement repris par Adorno dans son cours Ontologie und Dialektik et dans La dialectique négative, où il rend d'ailleurs hommage à Benjamin. Le monde ne s'invente pas sur la base d'un discours luminuex, comme le suggère la Bible - Au début fut le verbe - ou comme le voudraient les différents discours du devenir de l'être, d'un genre panthéiste comme le propose Spinoza. Le jargon heideggérien du Dasein, dans toutes ses phases de développement impérialiste, nazi ou encore antisémite de l'après guerre, se base sur une auto-proclamation identitaire de l'être qui refuse l'autre jusqu'à son extermination, une idée relayée par les fictions de Jünger ou encore les pamphlets de Sloterdijk qui se réfère explicitement à l'anti-humanisme d'Heidegger. Habermas a suffisamment approché ces discours de près pour s'en méfier, je pense. Vouloir définir le monde avec des mots fictionnels qui sont imposés aux autres, en dehors d'expériences et d'observations partagées, peut facilement conduire à des constructions propagandistes. Cela a pu déboucher sur cette analogie bancale entre Saddam Hussein et Adolf Hitler par laquelle Magnus Enzensberger a voulu justifier la guerre d'Irak en 1990. Alexander Kluge a très bien compris cet enjeu à sa manière, même s'il n'a pas pu s'empêcher d'écrire un récit fantasmagorique, où il envoie Adorno littéralement en enfer, le privant de la faculté de parler, le dépouillant de tous ces titres académiques et honorifiques, dans Le cinquième livre. C'est bien de la fiction, mais ce n'est pas tout le réel de la Théorie critique.

Adorno a une position sociologique et philosophique très claire : le travail conceptuel doit se mettre en relation avec l'expérience empirique du monde, dans un va-et-vient dialectique, pour ne pas succomber à un discours autoritaire de surplomb. Il élabore les conceptions et concepts de la Théorie critique à travers de grandes enquêtes empiriques, aux côtés de Fromm et Horkheimer notamment, à commencer par l'enquête sur les ouvriers allemands en temps de crise en 1930 qui prépare les études sur la personnalité autoritaire, suivi des observations et analyses des mass média et des enquêtes qui vont de l'après guerre à 1969, relancées ensuite par Brandt à Francfort et Negt à Hanovre à partir des années 1970, approches qui sont relancées aujourd'hui sur les cinq continents. Adorno fut le président de la société allemande de sociologie jusqu'à sa mort, et l'Institut de Francfort s'appelle officiellement Institut de recherche sociale / Institut für Sozialforschung depuis le début. Il s'agit d'organiser une dialectique entre l'expérience et le concept, dont la recherche empirique fait partie. Il ne faut surtout pas confondre le positivisme avec la recherche empirique. En réalité, le positivisme est peu étayé empiriquement, comme l'a aussi constaté le sociologue George Friedmann qui était en contact avec Adorno. Le texte programmatique où Adorno définit une recherche empirique qui ne serait ni positiviste, ni utilitariste ou administrative, mais critique, est le Memorandum qu'il adresse à Lazarsfeld aux Etats Unis en 1940. Globalement, la littérature, l'essai, l'élaboration conceptuelle critique et la recherche empirique qui accède à des expériences sensibles ne s'opposent pas, mais entrent en résonance dans la Théorie critique. Malheureusement, Habermas n'en a pas toujours tenu compte, ce qui a provoqué le jugement de Horkheimer en 1958, lorsqu'il affirmait que le jeune chercheur fait autant violence à la philosophie qu'à la sociologie. C'est une évaluation scientifique tout à fait exacte de dix pages, qu'on appelerait un pré-rapport dans l'argot universitaire actuel, que je n'ai pas cité dans mon 
livre pour ne pas attaquer trop frontalement l'oeuvre d'Habermas, sachant aussi à quel point le personnage est idéalisé dans l'espace francophone. J'ai eu tort sans doute.

\section{Timothée Moreau}

Bonjour Monsieur Neumann, la première question que je voudrais vous adresser concerne l'ensemble de votre démarche dans le livre qui nous occupe. Dès le titre, votre ouvrage se présente comme une charge théorique contre la pensée de Jürgen Habermas. Votre argumentation repose de façon générale sur les fondements idéologiques et méthodologiques défendus par l'auteur que vous mettez en regard, soit de positions issues de l'École de Francfort de la première génération, Theodor W. Adorno en tête, soit d'auteurs contemporains de Habermas (Axel Honneth ou Nancy Fraser par exemple). Vous critiquez l'origine libérale des concepts habermassiens, par exemple sa conception d'un espace public modelé sur l'Angleterre du XVIII ${ }^{e}$ siècle et de l'Allemagne de l'Ouest de l'après-guerre, c'est-à-dire d'un espace public bourgeois auquel vous semblez préférer la notion « d'espace public oppositionnel » dont Oskar Negt et, dans une moindre mesure, Alexander Kluge sont les référents principaux ${ }^{9}$. Vous analysez aussi de manière assez précise les origines théoriques des positionnements de ces différents auteurs, notamment les sources durkheimiennes de Jürgen Habermas qui semblent renforcer le caractère abstrait de sa théorie ${ }^{10}$. Votre ouvrage représente dès lors un apport tout à fait considérable dans le domaine de la sociologie contemporaine en ce qu'il permet de saisir les enjeux de positionnements théoriques et de les articuler entre eux.

Ce travail d'articulation critique des différents penseurs que je viens d'évoquer rend tout à fait explicite votre critique de la pensée habermassienne. Pourtant, cette dernière demeure centrale dans votre texte, si bien que le lecteur que je suis finit par s'interroger : quelle est, en définitive, votre position vis-à-vis de Jürgen Habermas? Ne voulez-vous lui reconnaître que le seul mérite de positions courageuses ou légitimes, un mérite de «bonne opinion" en quelque sorte? Ou bien trouvez-vous chez lui des ressources théoriques ou méthodologiques utiles à l'analyse de la situation actuelle? En exagérant un peu le propos, la question pourrait se poser dans des termes plus outranciers encore: quelle nécessité voyez-vous encore à vous référer à Habermas? Ne pourrait-on se contenter de construire une généalogie intellectuelle non classique qui, partant de la première génération de la Théorie critique aboutirait à des méthodes plus proches de celles de la micro-histoire par exemple? Permettez-moi de préciser cette première question.

18 À la première lecture de votre livre, on reconnaît dans l'introduction une attaque assez dure, dans le fond comme dans la forme, des positions de Habermas, à l'exception, me semblait-il, du paragraphe "Tournants et méandres $»^{11}$ qui portait au compte du philosophe quelques éléments positifs. À la relecture, il me semble plutôt déceler dans ce paragraphe une certaine ironie qui rend le propos d'autant plus critique à l'égard de l'auteur de La théorie de l'agir communicationnel. Par ailleurs, votre ouvrage ne peut être considéré comme une simple critique unilatérale de Habermas. En effet, vous reconnaissez aussi à ce dernier quelques mérites, notamment la «légitimité de ses critiques (envers le marxisme doctrinaire, la bureaucratisation étatiste, le souverainisme nationaliste, l'antisémitisme et la philosophie fasciste, en faveur d'une approche nonpositiviste, transdisciplinaire et cosmopolite) ${ }^{12}$ ». De même, en vous lisant, on peut porter au crédit du penseur l'apport du concept d'espace public qui, par ses difficultés et apories 
mêmes, a, en partie, permis l'émergence de concepts tels que celui «d'espace public oppositionnel ». Cette position vis-à-vis de Jürgen Habermas, plus nuancée que ce que n'annonçait en première lecture l'introduction de votre livre, me semble particulièrement intéressante, ne fusse que parce qu'elle justifie qu'il soit encore possible, voire nécessaire, de se référer à Habermas aujourd'hui. Votre position vis-à-vis de l'héritier le plus connu de la première génération de l'École de Francfort consiste-t-elle, en somme, à trouver dans sa conception de l'espace public le référent nécessaire pour la construction d'un modèle antagoniste, d'un espace public oppositionnel ${ }^{13}$ ?

Cette première question m'amène à une seconde, peut-être plus fondamentale, qui vise votre choix méthodologique. Si je vous lis bien, vous cherchez à proposer une sociologie qui puisse faire droit à l'expérience sensible ou vécue des individus et qui refuserait ainsi une vision théorique trop abstraite et totalisante. Vous vous référez dans plusieurs passages de votre ouvrage à des événements historiques et à des situations empiriques claires tout comme à des raisonnements théoriques et logiques précis. L'objectif serait de définir, si j'en crois cette phrase de votre conclusion, «la sociologie de Théorie critique par sa dialectique inimitable de l'expérience et du concept ${ }^{14} »$. Ma question est alors celleci : à quoi ressemble concrètement une telle méthodologie dialectique? S'agit-il de construire une sociologie faite d'événements et de vies singulières symptomatiques d'une époque ou d'une société? Renouez-vous par là avec une sorte de "paradigme du chiffonnier " comme on peut en trouver une esquisse chez des auteurs tels que Walter Benjamin ou Siegfried Kracauer ${ }^{15}$ (ou peut-être encore Erich Fromm, comme vous le suggére $\left.z^{16}\right)$, voire même, plus récemment, avec le paradigme indiciaire de Carlo Ginzburg ${ }^{17}$ et des partisans de la micro-histoire (à laquelle on pourrait peut-être lier Arlette Farge que vous citez ${ }^{18}$ ) ? Il s'agirait alors de considérer les expériences singulières comme les reflets ou la cristallisation d'une époque et de certaines franges de la société qui s'y développent. En d'autres termes, cette sociologie de Théorie critique que vous cherchez à définir, connaît-elle déjà des actualisations, même partielles, et si oui, sous quelles formes ? Cette question rejoint la première : la filiation particulière de la Théorie critique que vous tentez de forger n'est-elle pas un rappel des exigences méthodologiques de l'École de Francfort de la première génération, c'est-à-dire l'exigence d'une dialectique du théorique et du pratique?

Enfin, sous quelle forme un tel projet - de sociologie de Théorie critique - peut-il être mené et, surtout, exposé ? Voyez-vous dans la forme même des thèses, des questions et des critiques que vous formulez, une manière de mettre en mouvement une forme dialectique?

\section{Alexander Neumann}

21 Vos questions montrent que vous avec lu tout le livre avec attention, sans vous arrêter sur mon introduction volontairement provocatrice, qui est là pour donner envier de connaitre la suite, pour dynamiser le propos. Oui, c'est une charge critique à l'encontre d'Habermas, mais au fond mon intention est de le dépasser, de le délaisser même, au bénéfice d'une Théorie critique plus proche de l'héritage fondateur. J'ai découvert avec plaisir le livre de Muharren Acikgöz sur les différentes générations qui ont rythmé la Théorie critique, tiré d'une thèse germanophone dont le titre est Die Permanenz der kritischen Theorie. Habermas est parfois présenté comme la 3ème génération, alors que le disciple direct d'Adorno est plutôt Oskar Negt. Pour commenter cet héritage avec une 
sorte de clin d'oeil, Lucia Sagradini et moi-même avons crée une collection baptisé La Quatrième Génération.

Au sujet du projet d'une sociologie critique qui serait en capacité de danser le tango avec la pholosophie, à travers la Théorie critique, j'ai non seulement affirmé qu'il reste pensable, mais j'ai voulu retracer cela de manière très détaillée à partir des chantiers majeurs des Francfortois, puis dans des enquêtes moins amples que j'ai pu mener moimême avec les moyens du bord.

D'abord, j'ai reconstitué la portée des Etudes sur la personnalité autoritaire, amorcées par Fromm et Marcuse, ensuite animées par Adorno, Else Frenkel-Brunswik et d'autres, publiées en 1951 à la suite d'un rapport qui se base sur plus de deux milles personnes interrogées, mais qui n'est disponibles en langue française que depuis dix ans seulement. J'ai montré que la méthode particulière, raffinée et critique des Francfortois fut actualisée depuis l'après guerre en Allemagne, aux USA et ailleurs, et cela jusqu'à une date récente, apportant une lumière vive pour pénétrer les phénomènes autoritaires contemporains, dont la résurgence des mouvements fascistes. Globalement, les néofascistes américains ont parfaitement compris la force corrosive, intacte, de la Théorie critique, en la désignant comme le noyau du d'un "marxisme culturel", expression qui dérive du terme de "bolchévisme culturel" présent dans la propagande nazie de l'époque de Goebbels. De l'autre côté, la formation de nouveaux espaces publics oppositionnels, aux Etats Unis, en Europe du Sud, dans le Maghreb et ailleurs, se comprend plus facilement si l'on se réfère aux débats théoriques que Negt et Kluge ont mené envers et contre Habermas à partir de 1968, autour de l'espace public oppositionnel. Ces aspects appariassent dans le livre Après Habermas, dans des chapitres dédiés.

Dans un autre livre qui n'existe qu'en allemand, Kritische Arbeitssoziologie, réédité en 2016, j'avais aussi retracé comment la Théorie critique a réussi à saisir les phénomènes de l'automation, de l'informatisation ou de numérisation, de la précarisation et de la féminisation du monde du travail, notamment grâce au sucesseur direct d'Adorno à l'Institiut de Francfort, Gerhard Brandt, ou grâce aux travaux de Kern, Schumann et Negt. Je m'en suis servi dans mes enquêtes sociologiques à l'Institut für Sozialforschung de la Sarre pendant cinq ans. J'ai par exemple tenté de concrétiser cette approche critique dans des enquêtes et articles consacrés aux travailleurs transfrontaliers en Europe. Les travailleurs transfrontaliers ne dépendent d'aucune identité nationale et d'aucun Etat national en particulier, mais traversent un no man's land qui échappe à toute définition positiviste. Il semble presque logique que les rapports d'enquête sociologiques que j'ai rendu à différentes institutions européennes ont fini dans un tiroir ou comme papier à recycler. Penser avec Adorno que la catégorie du non-travail, le refus d'identification immédiate avec la circulation capitaliste, puisse ouvrir un espace d'émancipation m'invite aussi à regarder en face des phénomènes comme les Gilets jaunes, aujourd'hui, en 2018-19. Définir de ce qu'est ou de ce que veut un Gilet jaune est impossible au sens positiviste du terme, mais le phénomène montre au contraire que le non-identique indéfinissable provoque une ouverture, un décalage, un espace d'émanicaption possible, signalé par l'appel de Commercy.

Pour couronner le tout, j'ai fouillé les archives Adorno et Benjamin à Berlin, à la recherche de la formulation du concept originaire de Kulturindustrie ou industrie de la culture. Ce modèle critique est actuellement relancé pour démonter les nouveaux mass médias, comme Facebook ou la téléréalité à l'ère du président Trump, celui qui fut 
auparavant l'acteur d'une émission produite par exactement la même maison de production que celle qu'Adorno avait analysé dans les années 1950. Je vous invite à lire l'opulent article que j'en ai tiré, sur le site de la revue Variations. En paralèle, j'ai écrit quelques textes pour pointer comment la créolisation d'Edouard Glissant peut dialoguer avec la dielactique négative, y compris dans la mise en scène d'un ChaosOpéra.

Cette actualisation de la Théorie critique s'applique aussi au féminisme socialiste et international, à travers Nancy Fraser, Angela Davis, Axeli Knapp et Regine BeckerSchmidt, entre autres, sur le plan théorique et empirique, dont je rends hommage et que je vais développer encore dans un livre à venir. Disons rapidement qu'Enzo Traverso s'est trompé quand il a déclaré la fin de ce type de féminisme socialiste dans un récent livre.

Cette expansion des observations et discussions permet peut être de répondre à votre question bien balancée, qui est de savoir si'il est encore nécessaire de se référer à Jürgen Habermas aujourd'hui? La réponse est non, dit rapidement, pour ce qui me concerne. Cela ne veut pas dire du tout que son oeuvre serait nulle ou sans signification, bien au contraire. Habermas avait absolument besoin de se confronter à Marx, dans l'espoir de voler de ses propres ailes, même si l'exclusion de la critique marxienne l'a coupé de son aile gauche. Je pense personnellement que la contribution philosophqieu majeure d'Adorno, Dialectique négative, n'aurait pas été écrit avec une telle concision si son auteur n'avait pas ressenti la nécessité de contredire de manière éclantante le discours morbide de Martin Heidegger. A une échelle plus personnelle, je peux reconnaitre que la figure d'Habermas, que j'ai découvert au lycée à Düsseldorf où nous sommes nés à des époques différentes, m'a aidé à me familiariser avec l'univers de la Théorie critique, même s'il l'a lui-même rejeté dès 1971, année de ma naissance. Je possède encore un petit recueil de ses textes en format poche pour l'usage scolaire, à la couverture jaune. Les articles politiques d'Habermas sont beaucoup plus accessibles pour un adolescent en quête de sens que les livres majeurs d'Adorno ou les essais pointus de Benjamin, même si j'essayais d'en comprendre le sens au même moment. Habermas venait alors de contrer dans la presse des révisionnistes de droite autour de Nolte, qui voulaient briser le consensus antifasciste de l'après guerre, et je me suis spontanément retrouvé du même côté de la barricade antifasciste. Plus tard, à Paris, j'ai travaillé avec Jean-Marie Vincent ou Miguel Abensour, qui étaient les premiers introducteurs d'Habermas en France, et j'ai compris comment ils avaient très tôt salué l'effet anti-stalinien de sa pensée, qui fut réel dans une culture intellectuelle française profondément marquée par le marxisme soviétique ou ses avatars althussériens. Mais cet effet initiateur, critique, des premiers textes habermassiens s'est dispersé, pour se fondre finalement dans un mainstream postmarxiste aux penchants régressifs, après la chute du mur de Berlin. Jean-Marie Vincent m'avait mis franchement mis en garde contre les non-dits, impensés et apories du discours habermassien avant sa mort lorsque je rédigeais ma thèse. Lorsque j'ai rencontré Habermas de visu, en 2014, j'ai vu que cette figure d'autorité est indissociable d'une certaine posture autoritaire. Cet homme a la taille d'un géant et regarde ses critiques de loin. Puis, mon éditeur m'avait posé la question de savoir, en 2015, s'il fallait préciser en quatrième de couverture que Habermas était le principal théoricien vivant de la socialdémocratie européenne. Cela semblait aller de soi. Heureusement que nous ne l'avons pas marqué, puisqu'il s'est empressé d'abandonner ce qui reste de la social-démocratie peu de temps après, aux côtés d'Emmanuel Macron, contre l'avis de son propre parti, le parti social démocrate allemand. Disons que si le vide idéologique apparent sur lequel repose le macronisme n'est pas rempli par la doctrine démocrate de son supporteur Habermas, 
cette forme politique risque de dériver complètement vers un bonapartisme inconsidéré, sans médiation politique, alors que la théorie d'Habermas peut l'arrimer à une forme d'Etat de droit, si elle était prise au sérieux par l'exécutif. Voilà une valeur d'usage possible, dans le style des hégéliens de droite qui ne me ragrdent pas.

Grâce au travail de distanciation que m'a permis l'écriture d'Après Habermas, je me suis rendu compte à quel point cette théorie a dérivé vers les thèmes fondamentaux de la droite historique européenne, ce qui fait que je prends désormais au mot les critiques assez dures à l'encontre d'Habermas qui ont été formulés par Horkheimer. En tout cas, la réception d'Habermas semble désormais dangereusement indexé sur la côté de Macron, avec lequel il s'est suridentifié publiquement en France. J'ai vu que le nombre de citations francophones de son oeuvre sont en baisse depuis l'élection présidentielle, bien que les traductions de ses livres soient en hausse, bénéficiant d'une forte médiatisation dans la presse. Ce n'est peut-être qu'une simple coïncidence; analogie ne vaut pas explication. Il devient cependant palpable que son désinvestissement théorique et politique de l'espace de la gauche internationale le marginalise et muséalise, alors qu'il est encore bien vivant. Je préfère tout exposer maintenant, pour me taire au moment des hommages pour son 90ème anniversaire.

\section{Caroline Glorie}

Cher Alexander Neumann, en guise d'incipit aux questions que je voudrais vous adresser, j'aimerais commencer par la citation suivante tirée de Espace public et expérience d'Alexander Kluge et Oskar Negt: "L'espace public retrouve sa valeur d'usage lorsque l'expérience sociale s'organise en son $\operatorname{sein}^{19}$."

Partant de cette phrase, je voudrais vous interroger sur le concept d'espace public oppositionnel et, plus précisément, sur les spécificités de ce concept afin de pouvoir le distinguer d'un autre, celui d'opinion publique. Je ne pense pas pouvoir vous suivre dans l'usage du concept d'espace public oppositionnel que vous proposez dans votre livre Après Habermas. La théorie critique n'a pas dit son dernier mot. Les exemples que vous donnez recouvrent, il me semble, des expériences de diverses natures : tantôt vous citez, pour l'Allemagne, «la Révolution des conseils ouvriers [et les] délibérations au sein du mouvement syndical de l'après-guerre ${ }^{20}$ ", tantôt, pour la France, « la grève générale de juin 1936 et ses assemblées générales ». Vous donnez également des exemples plus récents : les « grèves générales au sein de l'Union européenne, [et le] printemps québécois " ou encore "un mouvement étudiant (Chili, Grande-Bretagne en 2012) ou une occupation de place publique (Syntagma, Puerta del Sol, Wall Street en 2012-2013) ${ }^{21} »$. Il me semble que la diversité des exemples que vous donnez ne permet pas de saisir la spécificité politique d'un espace public oppositionnel. Est-il d'avantage du côté de l'exceptionnalité politique ou d'avantage du côté de l'institutionnalisation du politique? Un espace public oppositionnel est-il reconnaissable parce qu'il rend possible un moment politique exceptionnel ? Et, si oui, quels sont les critères définitoires d'un tel moment ? Ou, à l'opposé, l'espace public oppositionnel est-il reconnaissable parce qu'il permet l'institution d'un moment politique ? Et, dans ce second cas, comment le distinguer d'un espace public bourgeois? Ces questions présupposent que l'espace public oppositionnel et l'espace public bourgeois ne peuvent pas être placés sur un même axe ou, pour le dire autrement encore : ils ne peuvent être les deux faces d'une même pièce. Ce postulat de départ fait fond sur deux conceptualisations différentes du concept de publicité : celle de 
Jürgen Habermas et celle proposée par Arlette Farge dans Dire et mal dire. L'opinion publique au XVIII siècle $^{22}$. Si, dans les exemples que vous donnez, il me semble difficile de reconnaitre la spécificité politique d'un espace public oppositionnel, c'est parce que la publicité d'un tel espace n'est pas clairement définie. Je ferai, pour ma part, l'hypothèse que la publicité d'un espace public oppositionnel ne peut pas être la même que celle d'un espace public bourgeois. Et ce qui va suivre convergera dès lors vers cette question très simple : quelles sont les spécificités de la publicité d'un espace public oppositionnel ?

31 Vous semblez faire de l'espace public oppositionnel et de l'espace public bourgeois deux modalités d'une même fonction de la publicité. Dans plusieurs de vos textes, vous insistez d'ailleurs sur le rapport de co-construction qui existe entre l'espace public bourgeois et

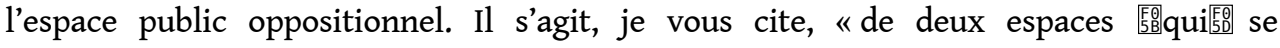
constituent l'un l'autre et évoquent deux dimensions de la société bourgeoise ${ }^{23}$ ». Vous insistez sur la dimension positive de cette co-construction: «Lorsque l'espace public oppositionnel s'épuise, l'espace public bourgeois régresse au profit de la fonction autoritaire de l'État, et lorsque l'espace public oppositionnel s'emballe, l'espace public bourgeois se déploie $e^{24} »$. Je pense qu'en faisant de ces deux espaces les deux faces d'une même pièce vous démontrez que la publicité de l'espace public oppositionnel et celle de l'espace public bourgeois fonctionnent sur le même mode.

Il me semble que cette indistinction nous expose au risque de réaliser une lecture habermassienne de Negt et Kluge. En réduisant l'espace public oppositionnel à un complément ou à un amendement du cadre conceptuel habermassien. Or, un des enjeux de la pensée de Negt et Kluge est la sortie du schéma de la représentation. Dès lors, comment comprendre la publicité pensée par ces deux auteurs? Je m'explique. Une première lecture de Espace public et expérience suggère l'existence d'une multiplicité d'espaces publics, propres à des groupes différents et engagés dans des rapports d'opposition par lesquels ils se façonnent mutuellement. Mais cette lecture ne permet pas de sortir du schéma habermassien de la représentation. D'ailleurs lorsque Habermas, dans "L'espace public, 30 ans après ${ }^{25}$ ", répond aux critiques qui lui ont été adressées, il insiste sur le fait qu'il ne remet pas en question sa pensée du fonctionnement de l'espace public comme espace de représentation. Habermas insiste justement sur le fait que, si le peuple est exclu de la sphère publique bourgeoise (et il reconnait l'existence d'une sphère public plébéienne), il participe, par son exclusion même, au fonctionnement hégémonique de la sphère publique bourgeoise :

Par son exclusion du pouvoir représentatif, le Peuple est ainsi inscrit dans les conditions mêmes de constitution de cette sphère publique représentative. Je pense comme auparavant que ce type de sphère publique [...] constitue l'arrière-plan historique des formes modernes de la communication publique ${ }^{26}$.

Dès lors, pour Habermas, mais pour nous également si nous n'établissons pas de distinction entre différents types de publicités, la multiplicité d'espaces publics n'empêche pas la domination absolue de l'un de ceux-ci parce que tous reposent sur le même principe de publicité : la représentation ${ }^{27}$.

Je voudrais maintenant pluraliser les conceptualités possibles de la publicité. À cette fin,

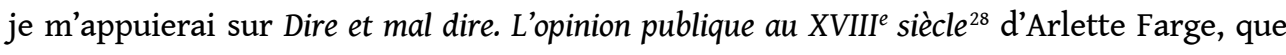
vous citez et qui fut écrit en réaction à l'Espace public de Habermas. Comme vous le rappelez dans votre livre, Arlette Farge montre, à partir de son travail dans les archives du XVIII ${ }^{\text {ème }}$ siècle de la ville de Paris, que "les couches populaires discutent à leur manière des affaires politiques du XVIII ${ }^{\mathrm{eme}}$ siècle ${ }^{29}$ ». Il n'y a pas seulement les bourgeois - 
classe alors en construction - qui discutent le pouvoir monarchique, il y a également le peuple. Farge prouve, contre Habermas, que le peuple - loin d'être inculte - discute, critique et se moque du pouvoir monarchique. Cependant, elle précise que bien qu'il ne s'agisse pas encore d'une "opinion publique populaire ${ }^{30}$ " (car le terme d'opinion publique est étroitement lié aux régimes de démocraties parlementaires), ces paroles du peuple sont, d'une certaine manière, construites par le pouvoir monarchique. En effet, l'analyse des archives révèle l'émergence et l'apparition d'un intérêt du pouvoir monarchique pour les avis du peuple; petit à petit se crée un rapport de co-construction entre les avis du peuple et le pouvoir monarchique :

Une curieuse spirale s'instaure, elle-même organisatrice de nouvelles figures d'expression populaire, même s'il s'agit bien sûr d'expressions non réglées des opinions. Sans existence ni statut, la parole populaire est un non-lieu politique en même temps qu'un lieu commun de la pratique sociale. Pourchassée par le pouvoir politique, elle prend forme et existence et s'élabore au cœur de ce système qui, contradictoirement, la nie et la prend en compte,

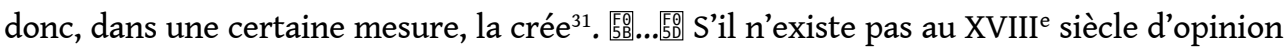
publique au sens moderne du terme, il existe des avis populaires dont les formes, les contenus et les fonctions évoluent à l'intérieur d'une posture monarchique qui leur donne vie tout en les refusant ${ }^{32}$.

Si nous connaissons aujourd'hui cette opinion de la rue du XVIII ${ }^{\text {ème }}$ siècle parisien, c'est précisément parce qu'elle a été écoutée, épiée et consignée par le pouvoir. On retrouve ici un geste foucaldien - geste dont Farge se réclame : le pouvoir crée le sujet qu'il gouverne.

Cependant, si le peuple du XVIII ${ }^{\text {ème }}$ siècle a bien l'intention de donner son avis, de juger, de chercher à savoir, il ne se constitue pas comme une force politique. S'il y a bien un rapport de co-construction entre les paroles populaires et le pouvoir monarchique, s'il y a contrôle permanent et réciproque de ces deux instances, on n'assiste pas, pour autant, à l'émergence d'une nouvelle figure du pouvoir. À cet égard, le cas de la bourgeoisie est différent puisqu'elle a su - en créant un espace public - devenir une force politique qui a fini par prendre la place du pouvoir monarchique. Le rapport que le peuple du XVIII ${ }^{\text {ème }}$ siècle entretient avec le pouvoir monarchique peut, en revanche, se comprendre comme une tension permanente, presque invisible, entre deux pôles, une tension qui finit par modifier ces deux pôles ${ }^{33}$. À force d'épier et d'enregistrer les paroles du peuple, le pouvoir monarchique perd sa propre maitrise. Il est pris dans un jeu qui le dessaisit de lui-même. Le pouvoir se trouve, pour une part, embarqué dans un jeu permanent (de basse intensité) avec le peuple. Ce dernier, quant à lui, vit une période prérévolutionnaire. En décrivant ces voix du peuple parisien, l'historienne donne donc forme à un ensemble de paroles qui préexistent à l'opinion publique (qui en sont la forme anticipative, l'opinion publique étant propre aux régimes de démocratie parlementaire). Ainsi, Farge permet de sortir de la dualité espace public bourgeois/espace public oppositionnel car elle identifie un troisième terme, l'opinion publique, à laquelle elle donne une généalogie.

Arlette Farge, tout comme Oskar Negt et Alexander Kluge, montre donc les limites du modèle habermassien. Si, d'une part, la monarchie entre en concurrence avec l'espace public bourgeois (et sera remplacée par ce dernier), les voix du peuple parisien préexistent à l'opinion publique. Ces deux « espaces » (espace public bourgeois et opinion publique) font un usage différent de la publicité : le premier fonctionne par la mise en publicité d'arguments rationnels tandis que le second fonctionne par un jeu de définition réciproque avec le pouvoir. De plus, si les avis du peuple inquiètent le pouvoir, la " publicité » des paroles populaires ne suffit pas à leur donner une force politique. 
40 Je voudrais suggérer que l'espace public oppositionnel se situe entre ces deux pôles. Il s'institue comme force politique, ce que ne fait pas l'opinion publique. Mais il ne peut pas fonctionner sur le même mode que l'espace public bourgeois, sinon il risque d'être absorbé par ce dernier (ou réduit à un amendement voire à une amélioration de l'espace public tel qu'il est conçu par Habermas). Dès lors, quelle est la publicité propre à l'espace public oppositionnel? Ajouter Farge à la discussion entre Habermas et Negt permet de mettre en perspective trois liens possibles différents entre le concept de publicité et celui d'espace public. Habermas instruit l'apparition d'un espace public bourgeois dont la publicité est fondée sur la discussion et l'argumentation rationnelles. Il tire, de son analyse historique, une norme : celle d'un modèle représentatif. Negt et Kluge pluralisent la notion de publicité. Farge, enfin, met en évidence un autre usage de la publicité lorsqu'elle décrit un espace qui est public et qui interagit avec le pouvoir sans s'instituer comme espace politique. Ces trois types de liens mis en perspective, pourriez-vous préciser le fonctionnement de la publicité d'un espace public oppositionnel? Lorsque vous décrivez les espaces publics oppositionnels, vous décrivez, entres autres, la Commune, les émeutes de 2006, les grèves dans les usines ou encore la colère générée par la crise de 2008. N'y a-t-il pas, parmi ces différents exemples, des manifestations différentes de la publicité ? N'êtes-vous pas en train de décrire tantôt des voix contestataires non constituées en espaces publics oppositionnels, tantôt des espaces constitués en tant que tel ? Quels seraient, selon vous, les critères identifiant la publicité d'un espace public oppositionnel?

\section{Alexander Neumann}

41 Votre référence au texte habermassien, L'espace public 30 ans après, me semble très pertinente, c'est probablement un point de non retour dans son parcours, si l'on le regarde rétrospectivement depuis son point d'aboutissement macronien. C'était sans doute un moment où il aurait pu tourner à gauche, juste après la chute de l'Union soviétique, alors que le stalinisme ne devait plus fournir de prétexte aux renoncements libéraux des gauches traditionnelles. Dans ce texte, il répond enfin sur le fond à ses critiques de gauche issues de 1968, au lieu de les traiter de fascistes de gauche, partant des arguments de Negt et Kluge, en passant par les travaux historiographiques d'E.P. Thompson ou d'Arlette Farge, les critiques féministes de Seyla Benhabib ou de Nancy Fraser qui, elle, n'est pas directement nommée. Oui, d'accord, concède-t-il, la plèbe aussi a pu s'exprimer politiquement à travers un espace public à certains moment, et les femmes ont joué leur rôle. Mais au lieu d'élargir son modèle de développement démocratique en ce sens, Habermas essaie de contenir et d'amoindrir les critiques qu'il juge lui-même légitimes, pour tenter de les ramener de force vers son schéma bourgeois initial. Par exemple, en réplique à l'historien E.P. Thompson il insiste que le mouvement ouvrier anglais, chartiste et travailliste reste à ses yeux une simple variante d'un libéralisme populaire. Ce concept reste flou et un seul coup d'oeil au mouvement Labour actuel, qui est devenu le principal parti de gauche de l'Europe depuis quelques années, arrimé aux mouvements syndicaux, socialistes et oppositionnels, montre à quel point cette définition est étriquée, inappropriée. Je n'ai vu personne définir le Labour actuel en tant que variante d'un libéralisme populaire, c'est un jeu de mots sans lendemain.

Une autre lecture reste toujours possible, celle d'un espace public bourgeois, représentatif et parlementaire, qui est forcé de tenir compte des mouvements sociopolitiques et oppositionnels qui le débordenet de manière cyclique, à travers la 
démocratie directe ou conseilliste. Nous avons encore eu la preuve de cette dialectique pendant l'hiver 2018 en France, ce que Habermas ne parvient pas à commenter, alors qu'il a désigné le pouvoir macronien comme un modèle. Le sous-titre allemand du livre de Negt et Kluge, où les deux auteurs avaient répliqué à l'ouvrage habermassien L'espace public, se nomme : Une dialectique des modes d'organisation des espaces public bourgeois et prolétarien. Cette dialectique se fait jour dès le début de la république allemande, d'abord en 1848, puis lors de la révolution des conseils de novembre 1918 qui débouche sur la première constituante. Habermas a toujours écarté ou occulté cette réalité historique, son modèle libéral qui se veut idéal-typique est en réalité une simple fiction philsosphique. Tous les historiens l'ont documenté, E.P. Thompson pour l'Angleterre et Arlette Farge pour le 18ème siècle français, où la prise de parole populaire joue déjà une grand rôle avant la révolution de 1789. Concernant le rôle des clubs et salons au 18ème siècle français, Antoine Lilti a précisé que la grande variété des salons s'inscrit dans un éventail qui obéit à une polarisation socio-politique, qui est mal résumé par le terme bourgeois. Habermas ignore à peu près tout de l'histoire sociale réelle, il ne reconnait ni le mouvement sansculotte dans les grandes villes, ni la révolution paysanne très ample, ni l'insurrection des jacobins noirs en outre-mer, et encore moins les premières formes féministes dont le clubs jacobins féminins. Vous avez peut-être raison que j'ai formulé ma critique d'une manière insuffisamment radicale, par souci du débat universitaire qui ne semblait pas encore prêt à recevoir la Théorie critique originale à haute dose.

Le caractère anhistorique et spéculatif de la philosophie habermassienne limite aussi la portée de son concept de publicité, qui est au départ défini par Kant, en appui de l'exemple vivant de la révolution française : Öffentlichkeit. Rendre public ce qui semblait auparavant une affaire personnelle appartenant à la société de cour. Ce concept politique tire toute sa signification des épreuves révolutionnaires qui fondent la démocratie, en 1789, en 1918, épreuves qui sont mis à jour dans les mouvements oppositionnels et enjeux actuels, mais la lecture habermassienne tend à isoler le concept de son contexte politique pour le rendre totalement abstrait. Son élève Honneth va jusqu'à supprimer la signification socio-historique de l'espace public bourgeois d'Habermas (bürgerliche öffentlichkeit), ne retenant qu'un espace public démocratique, un terme qui est vague et tautologique, dans la mesure où la publicité exprime la démocratie. C'est pour cela qu'un professeur francfortois, Gerhard Steinert, a qualifié la sociologie habermassienne de sociologie communale ou Kleinstadtsoziologie, adaptée à la vie sociale d'une petite ville où tout le monde se connait, discute et arrange les affaires, en dehors de toute analyse marxienne de la lutte des classes. Or, même les petites villes n'échappent pas au Gilets jaunes ou aux grèves cheminottes, qui existent aussi en Belgique.

\section{Jeremy Hamers}

Ma question concerne ce que je voudrais appeler ici le ou les langages des espaces publics oppositionnels et de ses acteurs dont, Caroline Glorie vient de le rappeler, vous soulignez la nécessaire multiplicité ${ }^{34}$. Ainsi, dans le premier chapitre de votre ouvrage, vous insistez longuement sur une distinction entre expérience sensible et expérience représentative. Permettez-moi de relire ici un des passages concernés :

Si l'ouverture de la démocratie représentative a rencontré ses limites, qui se manifestent dans les érosions ou débordements actuels, les tentatives historiques de traduire l'expérience vécue vers l'espace public par le moyen de la démocratie directe se heurtèrent à leur tour à des obstacles 
immanents dont le plus cité est sans doute la Commune de Paris. Ces deux principes délibératifs et organisationnels - représentatif et direct - interviennent aujourd'hui encore dans le débat au sujet de la représentation républicaine, de la démocratie participative et de l'espace public oppositionnel. Il apparait alors que la catégorie de l'expérience mérite d'être enrichie et différenciée en fonction de la polarisation qui se fait jour entre différents types d'espace public, représentatif, oppositionnel ou d'autres encore. L'apport théorique qui est proposé ici part de l'idée que les individus sociaux qui composent la société sont tous traversés par deux types d'expérience distincts, à savoir une expérience sensible (qu'Adorno qualifia de non-réglementée), et une autre modalité de l'expérience que l'on dira représentative, liée aux abstractions sociales ${ }^{35}$.

Dans Après Habermas, la distinction entre expérience sensible et expérience représentative - on dira, avec vous et à la suite d'Adorno, «normative » - vous conduit à penser la multiplicité des espaces publics comme étant à l'image des croisements de ces deux types d'expérience qui produisent des entités hybrides :

À mon sens, il est possible de penser les différents types d'espace public en tant que modes d'expression distincts de différentes dimensions de l'expérience qu'éprouvent les mêmes personnes. Tous les sujets des sociétés qui sont régis par l'échange marchand et l'État sont traversés par un principe abstrait d'une part et par un principe sensible d'autre part (il est possible que ces principes s'amenuisent parmi la grande bourgeoisie et des milieux autrefois résumés sous le terme de bohème). Il s'agit de deux principes opposés et distincts, mais complémentaires dans une même compréhension critique de la société. [...] Le principe abstrait concorde avec la répétition et la reproduction sociale, alors que l'expérience sensible engage la résistance et l'opposition. Au milieu de ces deux pôles, l'expérience se révèle souvent ambivalente, ce qui produit des formes publiques hybrides ${ }^{36}$.

48 L'expérience représentative concorde avec la "répétition et la reproduction sociale » dites-vous, tandis que l'expérience sensible "engage la résistance et l'opposition" (proches du « Protest » et du « Eigensinn » pensés par Negt et Kluge à la suite d'Adorno). Partant de cette distinction à laquelle vous associez directement aussi des questions d'expression, de dispositif, et j'ajouterais de «langage» (je songe notamment à votre propos concernant internet comme dispositif suscitant des modalités hybrides d'expression), je voudrais, à mon tour, revenir à un passage de Espace public et expérience de Negt et Kluge dans lequel on perçoit déjà une forme de complémentarité entre deux types d'expériences, mais dans une seule et même expression si je puis dire. Selon les deux penseurs, l'expérience sensible et l'expérience (ou compréhension) abstraite se rejoignent en effet dans ce que vous appelez "une même compréhension critique de la société ", pour aboutir à une expression commune :

Le besoin des travailleurs de s'orienter dans la société a souvent été décrit comme étant une conscience dichotomique. Pareille approche sociologique ne permet cependant pas de distinguer, d'une part, les différents composants de ce besoin fondamental et, de l'autre, les formes de l'espace public bourgeois qui les recouvrent. Ce qui est sous-tendu par ce besoin de proximité est une approche visant à recueillir une expérience immédiate et frontale, "car notre monde est humain, c'est-à-dire qu'il est le nôtre ». L'influence de l'espace public bourgeois se manifeste dans le fait que l'on scrute l'environnement pour y retrouver l'identité, simplement reconnaissable, des rapports marchands. Lorsque les travailleurs ne disposent pas de leurs propres modes d'expression pour articuler leurs intérêts, ils recourent à des stéréotypes que la société leur suggère. C'est le cas quand ils adoptent un clivage qui divise le monde en amis et ennemis. Le même procédé contient néanmoins une activité autonome: la tentative de saisir la réalité, telle qu'elle est. Cependant, même cette activité reste contradictoire, puisqu'elle ne fait qu'indiquer une voie juste pour 
appréhender la réalité tout en ayant recours à la simplification pour emprunter cette voie, donc à une vision du monde irréaliste et idéologique ${ }^{37}$.

Dans ce passage, les deux auteurs avancent que, dans certaines conjonctures sociales, il est inévitable que l'expression d'une expérience protestataire s'articule au langage de l'adversaire. Pour le dire vite : cette expression perd en autonomie car elle s'appauvrit au contact (et par la reproduction) de la norme imposée. Cet appauvrissement de l'expression est pourtant indissociable chez Negt et Kluge de « la tentative de saisir le réel tel qu'il est ", une tentative qui ancre la protestation dans un retour radical à l'expérience sensible. Partant de cette hybridation entre sensible et abstrait, entre protestation et reproduction d'une norme idéologique dans l'expression même de l'expérience, je me demande s'il est encore possible d'imaginer une expression protestataire qui ne serait pas hybride. Prenons un exemple concret qui déplace quelque peu mon propos mais qui permet aussi de l'expliciter : internet. En tant que potentiel support/outil d'un espace public oppositionnel aux yeux de certains, internet offre au sujet protestataire la possibilité de s'exprimer tout en lui suggérant d'emblée des modes d'expression qui inscrivent son expression dans une forme de reproduction sociale (logique de la visibilité, du succès quantifiable, de la formule courte ou illustrée, etc.). En tant que dispositif d'expressions multiples, internet réalise donc parfaitement l'ambivalence de toute expression tendanciellement émancipatoire identifiée par Kluge et Negt. À l'inverse de cet exemple, peut-on imaginer une forme d'expression de l'expérience sensible qui ne serait pas prise dans une tension dialectique entre l'autonomie et la norme? Et, le cas échéant, une expression non hybride, telle qu'Adorno a pu l'appeler de ses vœux, c'est-àdire une expression qui ne se corromprait pas au contact de son propre langage, pourraitelle encore servir une « tentative de comprendre la réalité telle qu'elle est »?

\section{Alexander Neumann}

51 Votre citation directe du texte d'Oskar Negt suscite en moi un effet de reconnaissance inattendu, alors que je l'ai traduit il y a des années. Ici, je ne vais pas défendre chaque argument de Negt à la manière d'un disciple académique, mais plutôt développer un point de vue. D'ailleurs, le livre de 1340 pages que Negt et Kluge ont co-écrit, Histoire et subjectivité rebelle, est tellement foisonnant d'idées, d'exemples et d'expériences qu'il ne serait pas simple d'en démontrer la cohérence conceptuelle définitive, ce n'est pas non plus leur objectif. Néanmoins, Negt cherche davantage la concision conceptuelle en philosophe que Kluge, qui assure le rôle créatif et iconoclaste débordant dans ce travail commun. Pour aborder vos deux questions, le rôle tâtonnant de l'expérience, puis la médiation de l'internet, le plus simple est peut être de passer par Adorno, qui avait d'ailleurs dirigé la thèse de Negt et orienté Kluge vers le cinéma en le mettant en contact avec Fritz Lang.

Mon point de vue est qu'Adorno appréhende les concepts par l'expérience, et interprète de manière critique les expériences à travers le concept. Le langage est loin d'être la seule médiation entre l'expérience et le concept. Il dit ainsi qu'un enfant, dont la langue maternelle serait l'allemand et qui n'a pas encore appris le français à l'école, peut saisir le sens d'un livre du Marquis de Sade. ${ }^{38}$ Il doit y avoir quelque expérience érotique, non-dite, qui l'éclaire dans son exploration des pages. Le concept même d'Eigensinn, subjectivité rebelle, se trouve non seulement dans la phénoménologie d'Hegel, mais déjà dans un conte pour enfant des frères Grimm, Das eigensinnige Kind, où un garçon entêté s'exprime encore après sa mort, sans mots, en levant le bras depuis sa tombe. La dialectique négative 
rappelle ces idées, c'est une réflexion qui se forge non seulement contre l'ontologie fermée d'Heidegger, mais encore à l'encontre de l'obsession philosophique d'Hegel qui cherche à canaliser toutes les expériences historiques et individuelles vers une conceptualisation explicite qui s'affirme finalement dans la souveraineté et l'Etat national. Pour prétendre que toute expérience soit résorbée dans et représentée par l'Etat, Hegel doit supprimer et occulter les expériences qui ne correpondent pas au schéma philosophique, mais ces expériences se vengent, s'expriment par d'autres voies, se lèvent tel un bras enfantin ou un soulèvement. D'autres expressions, parfois corporelles, parfois créatives, parfois violentes, parfois verbales, viennent alors déborder ou ré-interpréter les conceptualisations philosophiques et savantes. Le dictionnaire positiviste, le Littré, n'est alors d'aucun secours. Adorno a fait lui-même l'expérience que le cadre conceptuel et culturel d'une épouqe peut voler en éclat, d'abord comme jeune homme lors de la première guerre mondiale et de la révolution conseils allemande qui y met fin, puis lors de la victoire des nazis sous Hitler qui le contraint à l'exil, ou encore en 1968 peu de temps après la parution de La dialectique négative. A partir de 1940, l'interaction de la conceptualisation critique avec la recherche empirique d'un genre nouveau s'intensifie, sur fond d'expériences historiques absolument impensables jusque là. Ce qui n'empêche pas les Francfortois en exil de les penser, aux côtés d'autres chercheuses et chercheurs, afin de les nommer.

Ce genre d'apprentissage encourage une pédagogie alternative chez Negt, qui se souvient avec Freud que la construction d'une personnalité passe par une série d'abolitions partielles et par des ré-interprétations successives, à différentes étapes de la vie : enfance, adolescence, école, entrée de la vie active, découvertes sexuelles, épreuves ou deuils, crises, transmissions, confrontation à la mort.

L'expérience traverse un processus continu, souvent contradictoire, ce que Negt montre aussi chez les prolétaires et travailleurs. D'un côté, le travail vivant déborde les injonctions et représentations qui servent à mobiliser la force de travail sous la forme d'un vulgaire capital humain, dans le sens d'une production d'une vie qui comporte des aspects créatifs et utopiques. De l'autre côté, ce travail est obligé de s'inscrire dans les schèmes fétichistes du capitalisme, par l'obtention d'un salaire pour survivre et pour consommer, dans le cadre de la mondialisation capitaliste qui ne peut pas être simplement niée. Cette situation favorise des opinions et jugements stérotypées, qui ressemblent à des marques de voiture, de yaourt, ou à des titres de magazine, à une émission préférée. Le besoin de s'orienter encourage la personnification, l'identification à des tribuns qui parlent au nom des autres. Mais, lors d'un soulèvement, lors d'un passage à l'action et à l'acte, les prolétaires arrivent à discuter cette tension réelle, et on vient de voir que le discours économique abstrait du pouvoir est partiellement supplanté par la recherche d'une bonne vie, d'une bonne société, du vivre ensemble, de la réorganisation démocratique et de la répartition égalitaire des richesses. L'imaginaire médiatique et spectaculiare recule alors sous l'effet d'expériences et discussions plus directes. Jeremy Hamers : Nous vous remercions pour cette discussion passionnante. 


\section{NOTES}

1. Cette table-ronde a été organisée à l'Université de Liège le 11 décembre 2017 dans le cadre du séminaire mensuel de l'ARC GENACH (Genèse et actualités des humanités critiques - FranceAllemagne - 1945-1980) : http://genach.uliege.be/index.php/site/index

2. A. Neumann, Après Habermas. La Théorie critique n'a pas dit son dernier mot, Paris, Delga, 2015, p. 189.

3. «Quant au diagnostic de l'époque livré par la Dialectique de la raison, il n'a cessé de susciter des débats et des interprétations nouvelles, des plus élogieuses aux plus critiques, si bien que cet ouvrage demeure un centre de débats actuels. Cependant, en arrière-plan de l'analyse sociologique et historique, on a là la période de la Théorie critique la plus dépassée, peut-être justement parce qu'elle est fortement ancrée dans les affres de son contexte historique de rédaction, celui de la Seconde Guerre mondiale. À ce titre, hormis d'importants débats d'ordre philologique et moult conceptualisations encore pertinentes, le diagnostic sociologique d'ensemble porté par la Dialectique de la raison figure peut-être parmi les moins prometteurs pour penser l'avenir de la Théorie critique.» Voirol court ici le risque de reproduire ainsi la contradiction qui frappe bien des écrits récents contestant l'actualité de la Théorie critique : d'une part on lui reproche d'être dépassée parce que trop liée à une conjoncture historique bien précise, d'autre part on en dénonce son manque d'applicabilité à des objets concrets et contemporains. O. Voirol, «Quel est l'avenir de la théorie critique », Communications, 2012/1, n 21, p. 113-114.

4. A. Neumann, Après Habermas, p. 64. C'est aussi en substance ce que lui reproche Peter Sloterdijk lorsqu'il affirme, dans sa Critique de la raison cynique, que la Théorie critique est une pensée du retrait protectionniste visant à préserver la «sensibilité intacte» de l'intellectuel. P. Sloterdijk, Critique de la raison cynique [1983], trad. de l'all. par Hans Hildenbrand, Paris, Christian Bourgeois éditeur, 1987, p. 15.

5. Cette notion est librement définie à la suite de Guillaume Sibertin-Blanc et Stéphane Legrand :

G. Sibertin-Blanc, S. Legrand, Esquisse d'une contribution à la critique de l'économie des savoirs, Reims, Le Clou dans le Fer, 2008.

6. Ibid., p. 146.

7. Max Weber, Soziologie, Körner, 1973, p.193.

8. E. Kant, Von der Unmöglichkeit eines ontologischen Beweises vom Dasein Gottes (L'impossibilité d'une démonstration ontologique de l'existence - Dasein - de Dieu) in : Kritik der reinen Vernunft, Zweitausendeins, 2000, p.385.

9. A. Neumann, Après Habermas, plus particulièrement le troisième chapitre «Conceptualiser l'espace public oppositionnel ", p. 143 et suivantes.

10. Ibid., p. 66-72.

11. Ibid., p. 12-14.

12. Ibid., p. 19.

13. Ce qui semble être suggéré en p. 28.

14. Ibid., p.188.

15. Voir à ce sujet la préface d'Olivier Agard dans S. Kracauer, L'Ornement de la masse. Essais sur la modernité weimarienne, Paris, La Découverte, 2008, pp. 5-20, ainsi que O. Agard, Kracauer, le chiffonnier mélancolique, Paris, CNRS Éditions, 2010.

16. A. Neumann, Après Habermas, p. 59. 
17. C. Ginzburg, «Signes, traces, pistes. Racines d'un paradigme de l'indice », in Le Débat, 1980/6, n'6, p. 3-44.

18. A. Neumann, Après Habermas, p. 125.

19. O. Negt et A. Kluge, «Espace public et expérience » in O. Negt, L'espace public oppositionnel, trad. de l'all. et préfacé par A. Neumann, Paris, Payot, 2007, p. 57. Ce chapitre est constitué de deux chapitres de l'ouvrage d'O. Negt et A. Kluge, Öffentlichkeit und Erfahrung, Frankfurt-am-Main, Suhrkamp, 1972.

20. Ibid., p. 155.

21. Ibid., p. 195.

22. A. Farge, Dire et mal dire. L'opinion publique au XVIII siècle, Paris, Seuil, 1992.

23. A. Neumann, «L'espace public oppositionnel : lorsque l'oïkos danse à l'agora », Cahiers Sens public, 2013/1 (n¹5-16), p. 2.

24. Ibid.

25. J. Habermas reprend à son compte un geste foucaldien : «Foucault comprend les règles de formation des discours de pouvoir comme des mécanismes d'exclusion qui constituent chaque

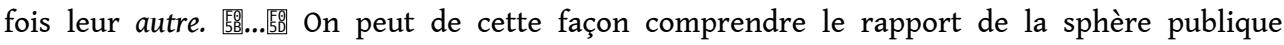
représentative du pouvoir traditionnel à la contre-culture refoulée du Peuple : le Peuple devait se manifester et s'exprimer dans une autre culture ». J. Habermas, "L'espace public, 30 ans après » in Quaderni, n¹8, Les espaces publics, Automne 1992, p. 161-191, ici p. 167.

26. Ibid.

27. La fin de l'article «L'espace public, 30 ans après » propose une réflexion sur la société civile, concept qui serait central, nous dit J. Habermas, si l'entreprise d'écriture de l'ouvrage L'espace public devait être recommencée aujourd'hui. L'auteur nous dit ceci : «Mais plus importantes encore sont les formes de diffusion et d'organisation, les modes d'institutionnalisation des supports d'un espace public non investi par le pouvoir ». Il est à remarquer que la société civile pourrait être une version habermassienne de l'espace public oppositionnel (notion sur laquelle il ne revient pas dans l'article). À tout le moins, il est certain que le schème de l'institutionnalisation joue un rôle central dans la mise en place d'un espace extrait des logiques de pouvoir. Ibid., p. 185. J. Habermas, L'espace public. Archéologie de la publicité comme dimension constitutive de la société bourgeoise [1962], trad. de l'all. par M. B de Launay, Paris, Payot, 1978.

28. A. Farge, Dire et mal dire.

29. A. Neumann, Après Habermas, p. 125.

30. A. Farge, Dire et mal dire, p. 14.

31. Ibid., p. 16-17.

32. Ibid.

33. Je remercie Antoine Janvier pour cette suggestion stimulante.

34. "J'ai ainsi suggéré de saisir et de définir l'espace public indépendamment de son appartenance à un groupe sociologique défini", mais plutôt comme «l'émancipation tendancielle d'une condition sociale initiale, et non pas comme la représentation politique directe d'un milieu social, bourgeois, ouvrier ou autre. » A. Neumann, Après Habermas, p. 25.

35. Ibid., p. 24.

36. Ibid., p. 27.

37. A. Kluge, O. Negt, « Espace public et expérience », p. 86-87.

38. Adorno, On parle français (en français et en italique dans le texte allemand) in : Minima moralia, Suhrkamp, 1959. 
INDEX

Mots-clés : Espace public, études post habermassiennes, modèles critiques, Jürgen Habermas, Theodor W. Adorno, Oskar Negt

\section{AUTEURS}

\section{CAROLINE GLORIE}

Groupe de recherche ARC GENACH - Genèse et actualités des humanités critiques - France-Allemagne 1945-1980, Faculté de philosophie et de lettres de l'Université de Liège 